\title{
Parameter identification and simulation of synchronous generator in an
}

\section{isolated power system}

\author{
$\mathrm{Pu} \mathrm{XIE}^{1, \mathrm{a}}$,Man $\mathrm{CAO}^{1, \mathrm{~b}}$ and Zhengchun $\mathrm{LIU}^{1, \mathrm{C}}$ \\ ${ }^{1}$ Mechanical Engineering College, Shijiazhuang 050003, China \\ axhpjhc2007@163.com, ’caoman724@163.com, nini82124@163.com
}

Keywords: Parameter identification; Synchronous generator; Isolated Power system; MATLAB

\begin{abstract}
The parameter estimation of a synchronous generator using the still frequency responses is presented in this paper. The required data is obtained to estimate the electrical parameters of the direct and quadrate axis models and the complete set of parameters was used in a five-order simulation model for a synchronous machine. To check the validity of the estimated parameters, the impact load simulation experiment is carried out at the generator terminals. A synchronous generator rate $120 \mathrm{kVA}, 400 \mathrm{~V}, 1500 \mathrm{rpm}, 50 \mathrm{~Hz}$ was used in the investigation. The measured data are compared with the simulation response of the generator model using the parameters estimated from the still frequency response, which can be seen that the data of simulation and the data of experimental match accurately.
\end{abstract}

\section{Introduction}

The isolated power system is different from the infinite grid, which is always supplied by the diesel generating set. The load characteristics of the isolated power system are different from that of the grid power supply system. Currently in the isolated power system, harmonic, pulse, impact load is increasing. Therefore, the dynamic characteristics of the isolated system are mainly determined by the interaction of the diesel generator and the critical load[1]. Synchronous generators play an important role in the stability of the power systems[2]. A proper model and accurate parameters for synchronous generators are essential for a valid analysis of stability and dynamic performance.

Currently, synchronous generator parameters in the simulation system of power system is used value provided by manufacturers or the typical value. Even a simplified model of synchronous generator is adopted because the lack of actual parameters.

In recent years, parameters of the synchronous generator model can be obtained by many tests which have been standardized[3,4]. Some tests are based on on-line measurements, while others are based on off-line tests. The sudden three-phase short-circuit is an online test while the frequency response is an off-line (or standstill) test[5]. The off-line tests can be carried out when the machine is not in service.

In this paper, the standstill frequency response, typically use low test voltages to obtain the resistances and unsaturated induction parameters is adopted to identify electrical parameter of synchronous generator.

\section{Model of the synchronous motor}

The objective of this paper is twofold. The first object is building the mathematical model of the synchronous generator. The second aim is to estimating the fundamental electrical parameters of the 
synchronous machine assumed model using the data acquired in the frequency response test.

Synchronous generator models have been developed since several decades ago. The set of differential equations, that describes the electrical dynamics of the generator, is based on a model structure defined by electrical equivalent circuits according to the two-axis theory. By transforming the three-phase variables, from the three-phase reference frame into a two windings reference frame, a simplification in the model is achieved [6,7].The mathematical model of the synchronous machine presented here used in the two loop rotor model based on the Park equation.

The equivalent circuit diagram of the $d$ and $q$ axis system can be obtained by the voltage and flux equations of the motor, as shown in Figure 1. The test procedure will be discussed according to the following equivalent,ircuit of $\mathrm{d}$ and $\mathrm{q}$ axis[8].

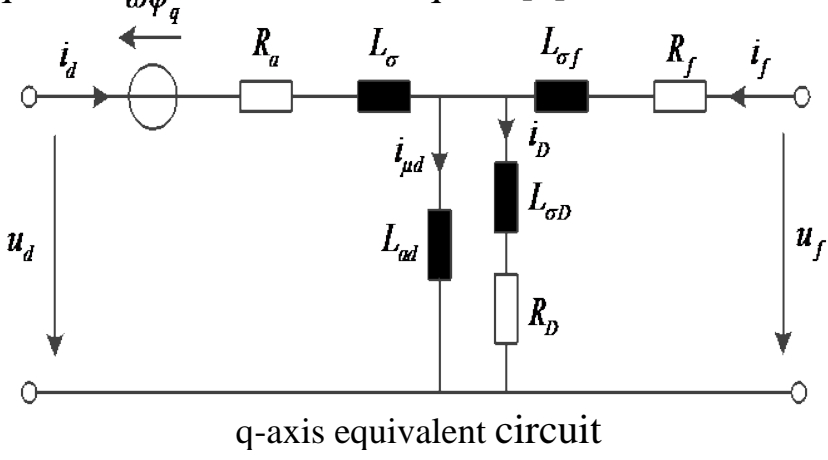

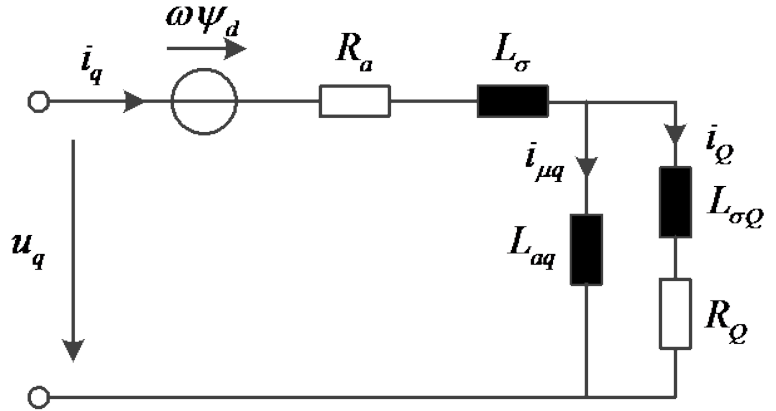

(b) q-axis equivalent circuit

Fig.1 Equivalent circuit

\section{Description of the frequency response experiment}

The parameters of the motor synchronous generator is obtained by frequency response method. The offline frequency response test has been intensively investigated and it has already been standardized[9]. Static frequency response method from the $\mathrm{d}$ axis and $\mathrm{q}$ axis flux and stator current and excitation voltage of the functional relationship, through a certain calculation to get the $d$ axis and q axis parameters.

The three-phase stator winding is excited by passing through a single phase variable frequency current, and the frequency response of the transfer function is obtained by collecting the voltage and current of the stator winding and the voltage and current of the excitation winding.

Tab.1 Measuring the transfer function

\begin{tabular}{|c|c|c|l|}
\hline $\begin{array}{l}\text { Operating impedance } \\
\text { of d axis }\end{array}$ & $\begin{array}{l}\text { Stator to excitation } \\
\text { transfer function }\end{array}$ & $\begin{array}{l}\text { Stator to excitation } \\
\text { transfer impedance }\end{array}$ & $\begin{array}{l}\text { Operating impedance } \\
\text { of q axis }\end{array}$ \\
\hline$Z_{d}(s)=\left.\frac{\Delta u_{d}}{\Delta i_{d}}\right|_{u_{f}=0}$ & $s G(s)=\left.\frac{\Delta i_{f}}{\Delta i_{d}}\right|_{u_{f}=0}$ & $Z_{a f o}(s)=\left.\frac{\Delta u_{f}}{\Delta i_{d}}\right|_{i_{f}=0}$ & $Z_{q}(s)=\left.\frac{\Delta u_{q}}{\Delta i_{q}}\right|_{u_{f}=0}$ \\
\hline
\end{tabular}

Static frequency domain method is based on the functional relationship between the $\mathrm{d}$ axis and $\mathrm{q}$ axis flux and stator current and excitation voltage, through a certain calculation to get the $\mathrm{d}$ axis and q axis parameters.

The principle of frequency response testing is applying on a certain frequency of disturbance signal on the tested system and recording its frequency response, and then through a certain calculation to get the $\mathrm{d}$ axis and $\mathrm{q}$ axis electrical parameters. 
The three-phase stator winding is excited by passing through a single phase variable frequency current, and the frequency response of the transfer function is obtained by collecting the voltage and current of the stator winding and the voltage and current of the excitation winding. A complete set of electrical parameters of a synchronous generator can be obtained from the four essential transfer functions, which is measured with the generator rotor stationary and can be seen in Table 1 .

Static frequency domain method is based on the functional relationship between the $\mathrm{d}$ axis and $\mathrm{q}$ axis flux and stator current and excitation voltage, through a certain calculation to get the $\mathrm{d}$ axis and q axis parameters. To obtain the desired frequency response (given in table 1), the rotor of the synchronous must be oriented according to certain requirements. And the excitation winding is either open or short circuit in the measurement process. After the test is connected well, the corresponding frequency of the sinusoidal current excitation is inputted on the selected frequency of each point of, and then the appropriate response output is captured and recorded. Finally, the corresponding data are processed by Matlab application program, and the discrete frequency points are sorted into corresponding frequency response curves. Static frequency response test plan is shown in figure 2 .

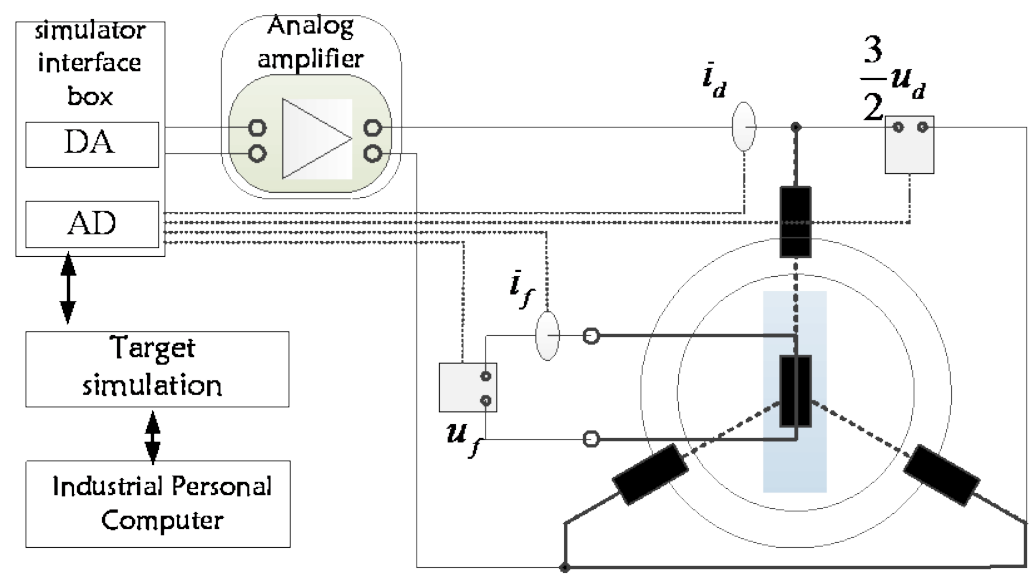

Fig. 2 Static frequency response test

\section{Estimation of fundamental parameters}

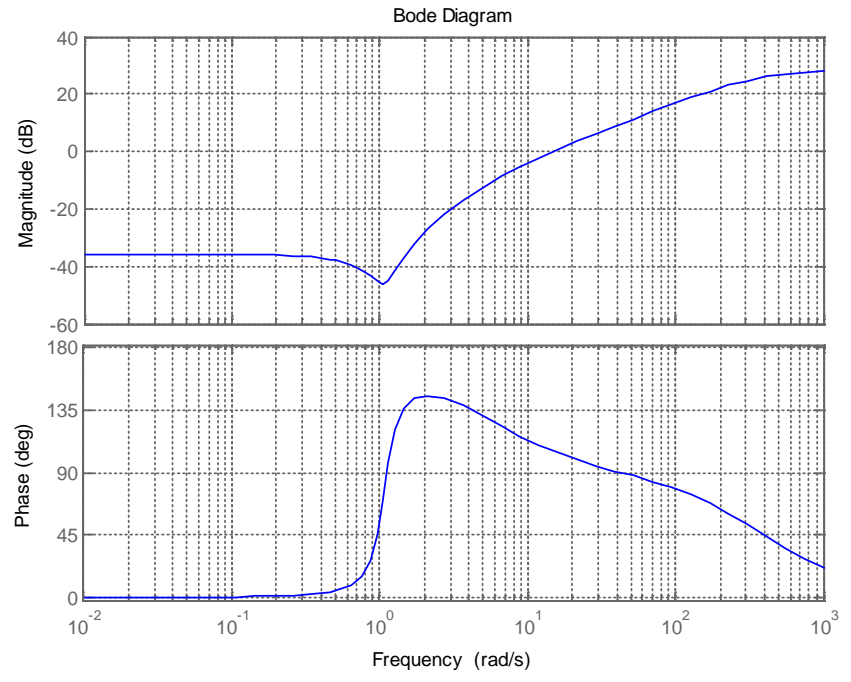

Fig.3. Curve of transfer function $Z_{\mathrm{d}}(\mathrm{s})$

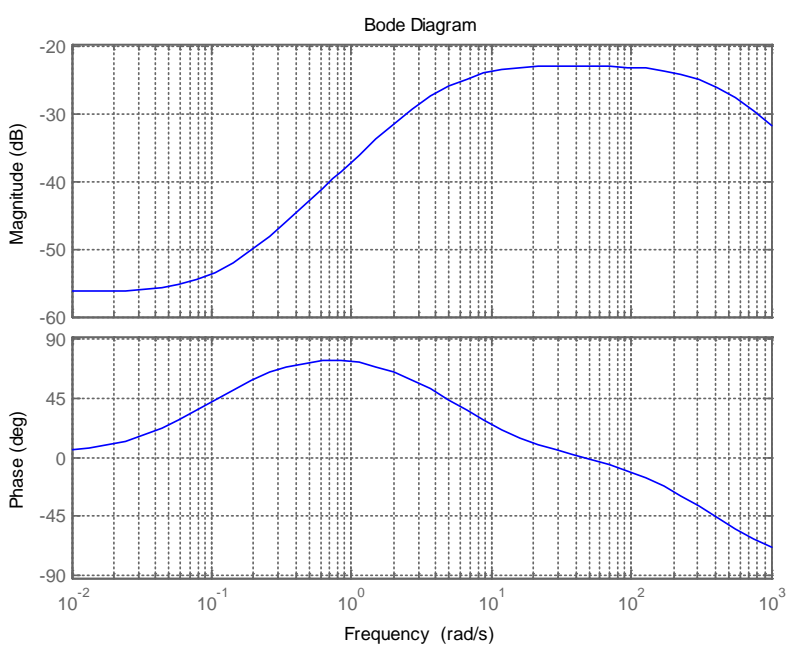

Fig.4. Curve of transfer function $L_{\mathrm{d}}(\mathrm{s})$

Figure 3 shows the operation of impedance frequency response curve. By drawing the curve of figure 4, we can determine the stator synchronous motor $\mathrm{d}$ axis inductance and the stator resistance. 
$u_{\mathrm{f}}=0$, namely the excitation circuit short circuit, type 1 can be obtained:

$$
\left.\frac{u_{d}}{i_{d}}\right|_{u_{f}=0}=R_{a}+j \omega L_{\sigma}+\frac{1}{\frac{1}{j \omega L_{a d}}+\frac{1}{j \omega L_{\sigma D}+R_{D}}+\frac{1}{j \omega L_{\sigma f}+R_{f}}}
$$

When the excitation frequency decreases to 0 , thus tends to $\mathrm{R}_{\mathrm{a}}$, therefore can determine the stator resistance.

$\lim _{s \rightarrow 0} Z_{d}(s)=R_{a}=0.0162 \Omega$

Subtract $\mathrm{Z}_{\mathrm{a}}$ from $\mathrm{Z}_{\mathrm{d}}$ and divide by the Laplace operator can get the operating inductance.

$$
\begin{aligned}
& L_{d}(s)=\frac{Z_{d}(s)-R_{a}}{s} \\
& L_{d}(s)=L_{\sigma}+L_{a d} \frac{1}{1+\frac{s L_{a d}}{s L_{\sigma D}+R_{D}}+\frac{s L_{a d}}{s L_{\sigma f}+R_{f}}}
\end{aligned}
$$

By the formula (3-3), the d axis stator inductance is equal to the value of the value at the frequency of 0 . The value of the amplitude frequency characteristic curve can be obtained.

$$
\lim _{s \rightarrow 0} L_{d}(s)=L_{d}=1500 \mu \mathrm{H}
$$

The method of using the frequency response curve data obtained from the test and the relation between the data and the frequency response curve obtained by the transformation is given here. After obtaining can be based on factory design data or experience to get a sense of leakage. Here, in the follow-up of the actual modeling and simulation to verify the rationality of the value. $L_{\sigma d}=74 \mu H$

List of other electrical parameters can also be obtained in the corresponding frequency curve, which will not go into here. The electrical parameters of generators as shown in table 2.

Table 2 Electrical parameters of generator

\begin{tabular}{|c|c|c|c|c|c|}
\hline$R_{a}(\Omega)$ & $L_{\sigma}(\mu \mathrm{H})$ & $L_{d}(\mu \mathrm{H})$ & $L_{a d}(\mu \mathrm{H})$ & $R_{D}(\Omega)$ & $L_{\sigma D}(\mu \mathrm{H})$ \\
\hline 0.0162 & 54 & 0.0015 & 0.001466 & 0.0624 & 74 \\
\hline$R_{f}$ & $L_{\sigma f}$ & $L_{q}$ & $L_{a q}$ & $R_{Q}$ & $L_{\sigma Q}$ \\
\hline 0.0090 & 116 & 692 & 638 & 0.0245 & 36 \\
\hline
\end{tabular}

\section{Verification of synchronous generator parameters}

After obtaining the motor parameters of the synchronous generator, the simulation model of the generator set is established in Matlab/Simulink, as shown in figure 5. A synchronous generator rate 120kVA, 400V, $1500 \mathrm{rpm}, 50 \mathrm{~Hz}$ was used in the investigation. The complete set of parameters was used in a machine model to simulate the impact load. The model is divided into three parts, which is the diesel engine, the excitation control and the generator model. 


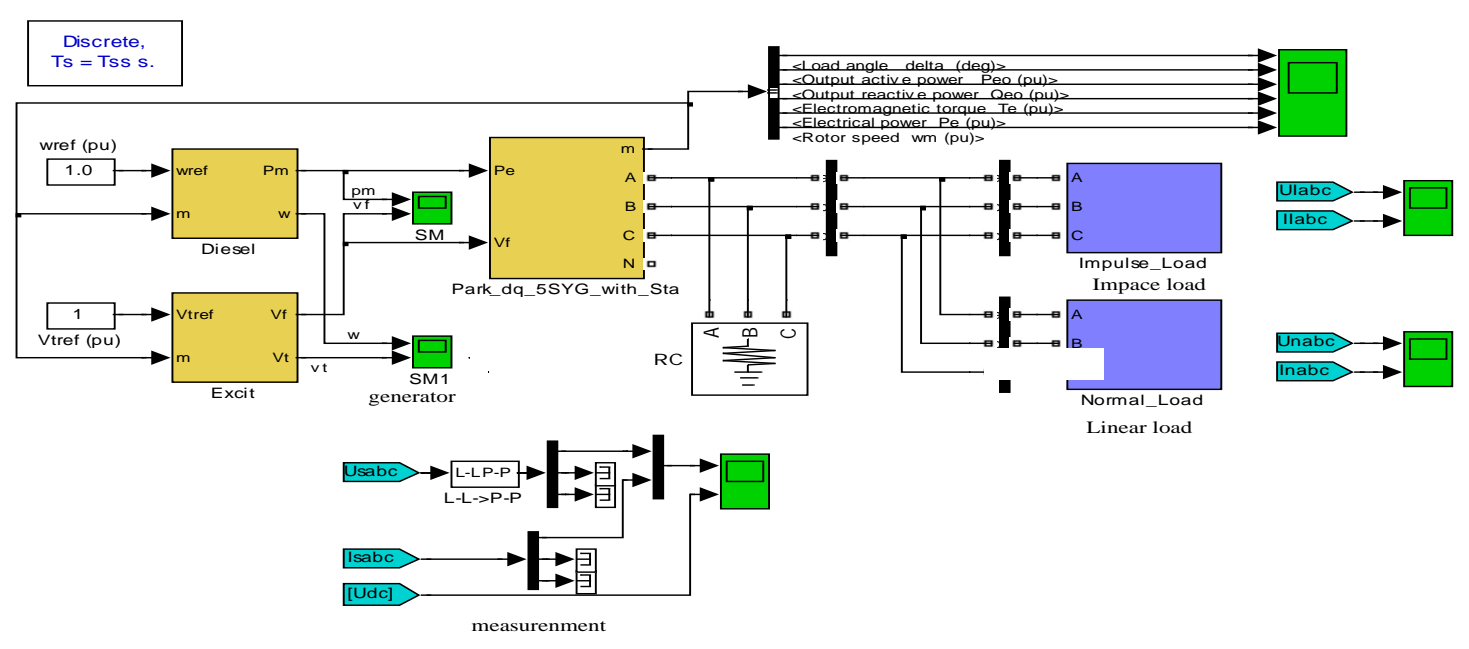

Fig.5 Verification model of electrical parameters

To check the validity of the estimated parameters, the impact load simulation experiment is carried out, which is shown in ${ }^{*} \quad$ e 5 . Based on the d-axis and q-axis equivalent circuits of the synchronous generator, its five-order parameter model is derived. In the simulation system, the impact load is used to simulate the transient high power demand characteristics of all kinds of devices for power supply in short time (within 10s). At the same time, the linear load in the loading and unloading process will cause the power supply bus instantaneous current and voltage fluctuation. The measurement model monitoring the voltage and current of AC bus and the voltage of DC side capacitor to verify whether the electrical parameters of the generator set is correct.

According to the above requirements, respectively to the different cases such as linear load in the loading and unloading, impact load start loading (full load, non full load), loading after startup (process load, uniform load) and unloading is simulated and analyzed. The model predictions were compared with measurements of the online test. The composition of loads of impact simulation test during loading the linear load is as following. Generator capacity is 60kVA, linear load is $p=20 \mathrm{~kW}, q=$ $4 \mathrm{~kW}$ and the load time is $2 \mathrm{~s}$. 


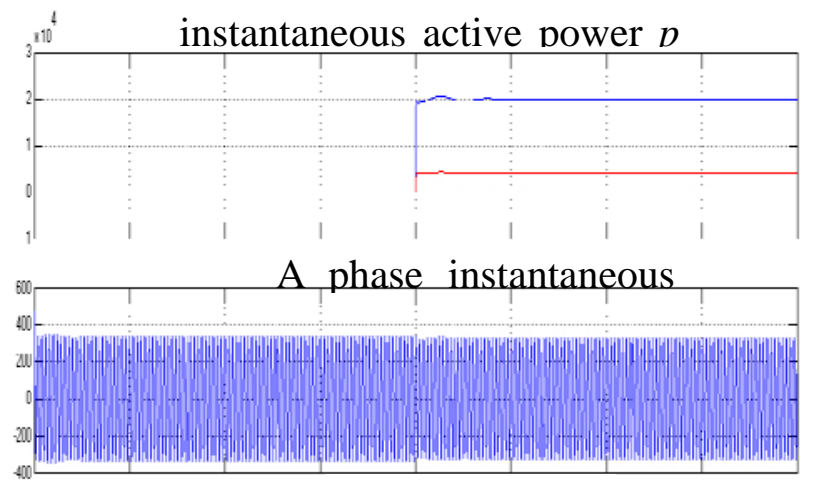

A phase instantaneous

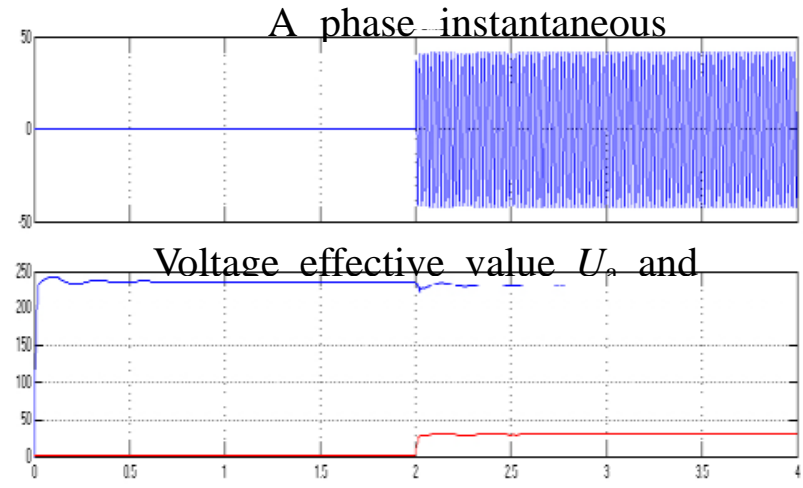

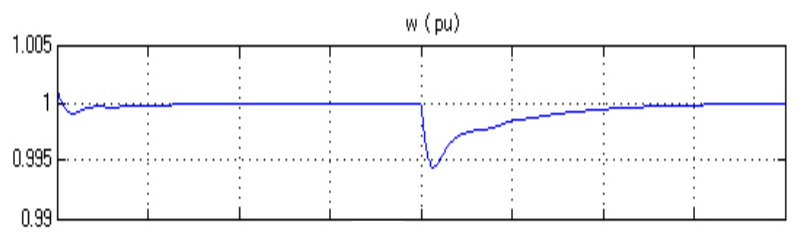

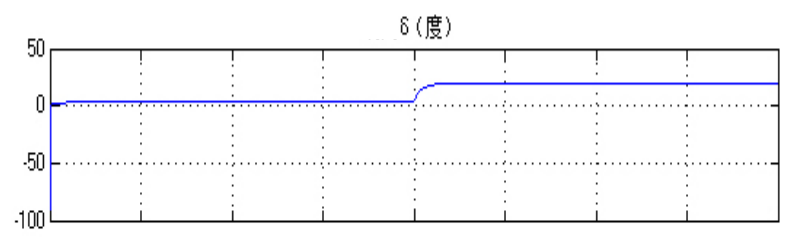

$\mathrm{Te}$
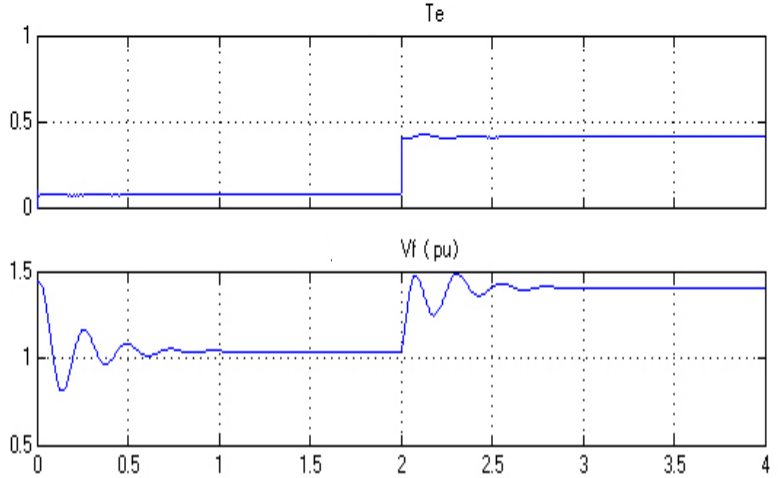

Fig. 6 The waveform of simulation

on

(a) electrical parameters

(b) parameters of generating units

The simulation waveform of instantaneous active power $p$, instantaneous reactive power $q, a$ phase instantaneous voltage $u_{a}$, instantaneous current $i_{\mathrm{a}}$, voltage effective value $U_{\mathrm{a}}$, current effective value $I_{\mathrm{a}}$, is shown in Figure 6(a). After loading, $p=20 \mathrm{~kW}, q=4 \mathrm{~kW}, u_{\mathrm{a}}$ is from $237 \mathrm{~V}$ to $230 \mathrm{~V}, \underline{i}_{\mathrm{a}}$ is from $0 \mathrm{~A}$ to $29.4 \mathrm{~A}$, the peak value $\underline{u}_{\text {apeak max }}=333 \mathrm{~V}, \underline{u}_{\text {apeak min }}=320 \mathrm{~V}$, the steady peak value $u_{\text {apeak }}=326 \mathrm{~V}$, and then fluctuation rate of voltage is $3.99 \%$. The generator internal parameters such as speed $w$, power angle $\delta$, electromagnetic torque $T_{\mathrm{e}}$ and excitation voltage $V_{\mathrm{f}}$ is shown in Figure 6 (b).

From Figure 6(b), it can be seen that the influence of the load on the internal parameters of the diesel generato.The drop of generator rotor speed $w$ is about $0.5 \%$, the time of fluctuations is $1.5 \mathrm{~s}$, power angle $\delta=20.25^{\circ}$, electromagnetic torque $T_{\mathrm{e}}=0.408 \mathrm{pu}$, excitation voltage $V_{\mathrm{fmax}}=1.5 \mathrm{pu}$, $V_{\text {fmin }}=1.25 \mathrm{pu}, V_{\text {fsteady }}=1.4 \mathrm{pu}, \triangle \mathrm{V}_{\mathrm{f}}=0.25 \mathrm{pu}$, fluctuation rate of excitation voltage is $17.9 \%$ and the time of fluctuate is $1.5 \mathrm{~s}$.

Sometimes the estimated parameters are compared with actual ones. The measured data are compared with the simulation response of the generator model using the parameters estimated from the still frequency response. A synchronous generator rate 120kVA, 400V, $1500 \mathrm{rpm}, 50 \mathrm{~Hz}$ was used in the investigation. The measured fluctuation rate of voltage is $3.94 \%$ and the measured fluctuation rate of excitation voltage is $17.6 \%$. It can be seen that the data of simulation and the data of experimental match accurately.

\section{Conclusion}

A model of the synchronous generator applied in an isolated power supply system is developed in this paper whose electrical parameters are obtained by still frequency responses. Furthermore, the 
description of the developed experimental for both standstill and online tests is presented. The developed model has been verified under the impact load simulation in the isolated power supply system. The measured data of online test are compared against the simulation response of the machine model using the parameters estimated from the still frequency response. Good accuracy was obtained and it was concluded that the set of parameters obtained from the still frequency response can predict complete direct and quadrate axis models for a synchronous machine.

\section{Acknowledgement}

This paper is supported by National Natural Science Foundation of China (No. 51307184).

\section{References}

[1] Shi Wei-fang, Zheng Hua-yao. Marine Automatic electric power station system simulation[[J]. Journal of system simulation, 2003,15(9):1249-1252.

[2] Li Dong-hui, Zhang Jun-dong, He Zhi-bin. Modeling and Simulation of the power system on practice teaching ship [J]. Journal of Shanghaijiaotong university，2008，42(2):190-193

[3] IEEE guide for synchronous generator modeling practices and applications in power system stability analyses. Park Avenue (NY): IEEE; 2002.

[4] IEEE guide: test procedures for synchronous machines. New York (NY): IEEE;1995.

[5] Test guidelines for synchronous unit dynamic testing and model validation. Salt Lake City (UT): Western Systems Coordinating Council; 1997.

[6] Aliprantis DC, Sudhoff SD, Kuhn BT. A synchronous machine model with saturation and arbitrary rotor network representation. IEEE Trans Energy Convers 2005;20(3):584-94.

[7] I.M. Canay. Causes of Discrepancies on Calculation of Rotor Quantities and Exact Equvalent Diagrams of the Synchronous Machine. IEEE Trans PAS-88.

[8] Tumageanian A, Keyhani A. Identification of synchronous machine linear parameters from standstill step voltage input data. IEEE Trans Energy Convers1995;10(2):232-40.

[9] Dehghani M, Karrari M, Rosehart W, Malik O. Synchronous machine model parameters estimation by a time-domain identification method. Int $\mathrm{J}$ Electr Power Energy Syst 2010;32(5):524-9. 